\title{
The Efficacy of ${ }^{18} \mathrm{~F}-\mathrm{FDG}$ PET/CT in Detecting Colorectal Cancer Recurrences
}

\author{
(1) Aysun Küçüköz Uzun'1, (1) Tülay Kaçar Güveli², (D) Filiz Özülker³ , (1) Tamer Özülker³ \\ 1 University of Health Sciences Turkey, Kartal Dr. Lütfi Kırdar City Hospital, Clinic of Nuclear Medicine, Istanbul, Turkey \\ 2 University of Health Sciences Turkey, Istanbul Bakırköy Dr. Sadi Konuk Training and Research Hospital, Clinic of Nuclear Medicine, istanbul, Turkey \\ 3University of Health Sciences Turkey, Prof. Dr. Cemil Taşcıoğlu City Hospital, Clinic of Nuclear Medicine, İstanbul, Turkey
}

\section{Abstract}

Objective: Early diagnosis of recurrent colorectal cancer (CRC) has an impact on the patient's survival and quality of life. This study aimed to determine the efficacy of ${ }^{18} \mathrm{~F}$-fluorodeoxyglucose $\left({ }^{18} \mathrm{~F}-\mathrm{FDG}\right)$ positron emission tomography/computed tomography (PET/CT) imaging in restaging patients with previously operated CRC, with elevated tumor markers and/or suspicious, equivocal, or pathological conventional imaging modality findings.

Methods: Data of 75 patients (42 males, 33 females) with CRC were retrospectively analyzed. All patients had undergone an operation, with suspicion of recurrence because of elevated tumor markers and/or equivocal or pathological conventional imaging modality findings during follow-up. Only patients, who have undergone conventional imaging methods (CIM) (e.g., CT and magnetic resonance imaging) and/or with serum tumor marker (carcinoembryonic antigen) measurements done within the first month preceding the PET/CT scan, were included in the study.

Results: Recurrence was confirmed in 58 patients with the clinical follow-up or pathological findings after the ${ }^{18} \mathrm{~F}-\mathrm{FDG}$ PET/CT imaging. The sensitivity, specificity, accuracy, and positive and negative predictive values of ${ }^{18} \mathrm{~F}-\mathrm{FDG} \mathrm{PET} / \mathrm{CT}$ in the diagnosis of CRC recurrence were $93.1 \%$, $88.2 \%, 92 \%, 96.4 \%$, and $78.9 \%$, respectively. The ${ }^{18} \mathrm{~F}-\mathrm{FDG}$ PET/CT was superior to CIM and serum carcinoembryonic antigen (CEA) measurements in detecting recurrent diseases in patients with CRC.

Conclusion: The ${ }^{18} \mathrm{~F}-\mathrm{FDG}$ PET/CT imaging was efficient for early detection of patients with recurrent CRC having elevated serum CEA levels. The overall success of the ${ }^{18} \mathrm{~F}-\mathrm{FDG}$ PET/CT in detecting recurrences in patients with elevated CEA levels and negative, equivocal and/or pathological findings of conventional imaging modalities was 70.6\%. PET/CT exhibited a 93\% success in detecting the recurrence in cases referred to ${ }^{18} \mathrm{~F}-\mathrm{FDG}$ PET/CT depending on suspicious findings on CIM and/or elevated CEA results. In our study, the ${ }^{18} \mathrm{~F}-\mathrm{FDG}$ PET/CT has changed the disease management in $33.3 \%$ of patients.

Keywords: Colorectal cancer, recurrence, metastasis, positron emission tomography, carcinoembryonic antigen

\section{INTRODUCTION}

Colorectal cancer (CRC) is the third most common cancer both in men and women and comprises $10 \%$ of all cancers (1). Despite radical surgery combined with chemotherapy and radiotherapy, the recurrence rate is $40-50 \%(2,3)$. Recurrences are most commonly localized to a single organ, such as the liver, or an anatomic region, such as the pelvis $(4,5)$. The outcome of CRC has significantly improved with the introduction of metastasectomy for isolated liver involvement treatment and effective chemotherapeutic agents (6-8). Surgery for singlesite was reported as $23-27 \%$ in the $5-y$ disease-free survival $(4,5)$. Therefore, early diagnosis of the recurrent disease has an impact on the patient's survival and quality of life. Before the introduction of ${ }^{18} \mathrm{~F}$-fluorodeoxyglucose $\left({ }^{18} \mathrm{~F}-\mathrm{FDG}\right)$ positron 
emission tomography/computed tomography (PET/CT), postoperative evaluation of patients with CRC was done by physical examinations, colonoscopy, and conventional imaging methods (CIM), such as ultrasound, $\mathrm{CT}$, and magnetic resonance imaging (MRI), to confirm recurrence, especially with increased carcinoembryonic antigen (CEA) levels. These techniques had limitations in distinguishing between postsurgical anatomic changes and scarring after radiation and residual or recurrent disease (9). The ${ }^{18} \mathrm{~F}-\mathrm{FDG}$ PET/CT was a widely accepted imaging method in the management of a variety of neoplastic diseases, including CRC. Many studies demonstrated the value of the PET in the diagnosis of CRC recurrence in the postoperative period $(10,11)$. The present study retrospectively evaluates the efficacy of ${ }^{18} \mathrm{~F}$-FDG PET/CT in the restaging of patients who were previously operated on due to CRC, with elevated CEA levels, and/or suspicious, equivocal, or pathological CIM findings.

\section{METHODS}

\section{Patients}

A total of 75 patients, wherein 42 were male and 33 were female, with a mean age of 62.1 years (range 30-82 years), suspected with recurrent CRC, who underwent PET/CT scanning from September 2007 to April 2009, were retrospectively reviewed. All patients had undergone an operation and presented with a suspicion of recurrence because of elevated tumor markers and/or equivocal or pathological CIM findings during follow-up. Only patients who had undergone CIM (CT, MRI) and/or had serum tumor marker (CEA) measurements done within the first month preceding the ${ }^{18} \mathrm{~F}-\mathrm{FDG}$ PET/CT scan were included in the study.

The ${ }^{18} \mathrm{~F}$-FDG PET/CT scan results, conventional imaging studies, and CEA levels were compared with the histopathological findings or follow-up results for 6-19 months.

This study was carried out with the approval decision of Okmeydani Training and Research Hospital Clinical Research Ethics Committee dated April 20, 2009, and numbered 260. Consent was obtained from all patients who were scanned.

Demographic details of patients are presented in Table 1.

\section{PET/CT Scan}

PET/CT studies were carried out using an integrated PET/CT scanner, consisting of a full-ring HI-REZ LSO PET and a six-slice CT (Siemens Biograph 6; Siemens, Chicago, USA). Patients were instructed to fast for at least $6 \mathrm{~h}$ before the ${ }^{18} \mathrm{~F}$-FDG injection. Blood glucose levels were measured before the study and ${ }^{18} \mathrm{~F}$-FDG injections were given only when the blood glucose levels were below $11.11 \mathrm{mmol} / \mathrm{L}$. Patients were injected with 296-555
$\mathrm{MBq}$ of ${ }^{18} \mathrm{~F}-\mathrm{FDG}$ according to body weight. Whole-body imaging commenced $60 \pm 5 \mathrm{~min}$ after the ${ }^{18} \mathrm{~F}$-FDG injection. The CT portion of the study was done without an intravenous contrast medium, just to define anatomical landmarks and make attenuation corrections on PET images. CT was first acquired with the following parameters: $50 \mathrm{mAs}, 140 \mathrm{kV}$, and 5-mm section thickness. Whole-body CT was performed in a craniocaudal direction. PET images were acquired in a three-dimensional mode, from the base of the skull to the mid-thigh, with 5-7 bed positions of $3 \mathrm{~min}$ each, and PET data were collected in a caudocranial direction. The cranium was also included if any known or suspicious brain metastasis were observed. After the acquisition was over, a quick evaluation of images was done and additional regional delayed spot images were obtained in cases with suspicious ${ }^{18} \mathrm{~F}$-FDG uptake especially at the colon segments. The CT data were matched and fused with the PET data.

\section{Image Analysis}

PET images were visually interpreted by three experienced nuclear medicine physicians about PET/CT fusion and CT images. Differences were settled by consensus reading. Positive PET findings were defined as the focal accumulation of ${ }^{18} \mathrm{~F}-\mathrm{FDG}$

\begin{tabular}{|l|l|}
\hline \multicolumn{2}{|l|}{ Table 1. Demographic details of patients } \\
\hline Characteristics & 75 \\
\hline Total number of patients & 62.1 \\
\hline Age (years) & $30-82$ \\
\hline Mean & \\
\hline Range & 42 \\
\hline Gender & 33 \\
\hline Male & $25.7 \pm 22.1(3-118)$ \\
\hline Female & \\
\hline $\begin{array}{l}\text { Time from the initial diagnosis to recurrence } \\
\text { (months) }\end{array}$ & \multicolumn{2}{|l|}{} \\
\hline Number of patients according to tumor localizations \\
\hline Ascending colon and cecum & $15(20 \%)$ \\
\hline Transverse colon & $2(3 \%)$ \\
\hline Descending colon & $7(9 \%)$ \\
\hline Sigmoid colon & $4(5 \%)$ \\
\hline Rectosigmoid & $9(12 \%)$ \\
\hline Rectum & $38(51 \%)$ \\
\hline Tumor histopathology & $70(93 \%)$ \\
\hline Non-mucinous adenocarcinoma & $10(14 \%)$ \\
\hline Well-differentiated & $53(76 \%)$ \\
\hline Moderately differentiated & $7(10 \%)$ \\
\hline Poorly differentiated & $5(7 \%)$ \\
\hline Mucinous adenocarcinoma & \multicolumn{2}{|l|}{} \\
\hline
\end{tabular}


above the normal level of surrounding tissue, excluding the physiologically increased uptake.

\section{Statistical Analysis}

All obtained data in the study were recorded in the Microsoft Office Excel 2003 for Windows program. Statistical Package for the Social Sciences 16.0 for Windows was used for statistical evaluation and analysis. Data are expressed as means \pm standard deviation. Sensitivity and specificity, as well as positive and negative predictive values [(PPV and NPV), respectively] and accuracies of the ${ }^{18} \mathrm{~F}-\mathrm{FDG}$ PET/CT, CIM, and CEA measurements, were determined.

\section{RESULTS}

Of the 75 patients, 37 (49\%) had colon cancer and 38 (51\%) had rectal cancer. A recurrence was eventually diagnosed in 58 of 75 patients (77.3\%). The duration between the treatment (surgery and/or chemotherapy and/or radiotherapy) and the detection of recurrence in these patients was 25.7 months on average (range 3-118 months). Of the 75 patients, 30 (40\%) patients diagnosed with recurrence were histopathologically confirmed following surgery or biopsy and $45(60 \%)$ patients were confirmed with results from follow-up. Of the 75 patients referred to us with the suspicion of recurrence, 19 (25\%) had elevated CEA levels, 24 (32\%) had only suspicious CIM findings, and 29 (39\%) had both. Three patients (4\%) were referred to ${ }^{18} \mathrm{~F}-\mathrm{FDG}$ PET/CT scan since the complaints and physical findings raised suspicion of recurrence even without marker levels and CIM finding abnormality.

Serum CEA levels were above normal in 48 (64\%) patients and within the normal range in 27 (36\%) patients. CIM findings were pathologically positive or suspicious for pathology in 53 (71\%) patients and without any abnormality in the remaining 22 (29\%) patients.

Among the 87 confirmed metastatic lesions detected in 58 patients with recurrence, 23 local recurrences, 24 liver metastases, 13 lung metastases, 9 serosal implants in the abdomen, 8 lymph node metastases in the abdomen, 5 bone metastases, 4 mediastinal lymph node metastases, and 1 peritoneal carcinomatosis were found.

Of the 58 patients with recurrence, ${ }^{18} \mathrm{~F}$-FDG PET/CT accurately detected the lesion in 54 patients. Of the 17 patients who were proven to have no metastasis, the ${ }^{18} \mathrm{~F}-\mathrm{FDG}$ PET/CT was truly negative in 15 patients. The recurrent CRC evaluation revealed sensitivity, specificity, accuracy, PPV, and NPV of ${ }^{18} \mathrm{FDG}$ PET/CT of 93.1\%, 88.2\%, 92\%, 96.4\%, and 78.9\%, respectively.
Of the 48 patients with above-normal serum CEA levels, the recurrent disease was confirmed in 42 patients. In 6 patients who were proven to have no metastatic disease, serum CEA levels were above normal, leading to false-positive results. Of the 27 patients with normal CEA levels, 11 had no recurrent disease, whereas 16 had recurrent disease. Therefore, the sensitivity, specificity, accuracy, PPV, and NPV of serum CEA levels were calculated as $72.4 \%, 64.7 \%, 70.6 \%, 87.5 \%$, and $40.7 \%$, respectively.

Of the 75 patients included in the study, CIM was reported suspicious, equivocal, and pathological in 53 patients. The CIM detected the recurrence correctly in 45 of these 53 patients, but lesions were reported as positive in 8 patients, although with no recurrent disease. Among the 22 patients who were evaluated as normal on CIM, 9 had no recurrent disease, yielding truenegative results, whereas 13 patients were reported as normal, which led to false-negative results. The recurrent evaluation calculated the sensitivity, specificity, accuracy, PPV, and NPV of CIM as $77.5 \%, 52.9 \%, 72 \%, 84.9 \%$, and $40.9 \%$, respectively.

The liver was the site of metastasis in 24 of 58 patients with recurrent disease. The sensitivity, NPV, and accuracy of ${ }^{18} \mathrm{~F}-\mathrm{FDG}$ $\mathrm{PET} / \mathrm{CT}$ in the evaluation of hepatic metastases were $100 \%, 100 \%$, and 95\%, respectively. Similarly, the serum CEA levels in the 24 patients with liver metastases were elevated showing sensitivity, NPV, and accuracy of $100 \%, 100 \%$, and $85 \%$, respectively. The CIM were true-positive in 19 of 24 patients and false-negative in 5 , yielding a sensitivity of $79 \%$ and accuracy of $68 \%$.

Local recurrences were found in 23 of 58 patients with recurrent disease. The sensitivity, NPV, and accuracy of the ${ }^{18}$ F-FDG PET/ CT in the evaluation of local recurrence were $100 \%, 100 \%$, and $95 \%$, respectively. The serum CEA levels were elevated in 17 of 23 patients with local recurrences and gave false-negative results in 6 patients; showing a sensitivity, specificity, and accuracy of $73.9 \%, 64.7 \%$, and $70 \%$, respectively. Meanwhile, the CIM yielded true-positive results in 16 of 23 patients and false-negative results in 7 patients resulting in sensitivity, specificity, and accuracy of $69.5 \%, 52.9$, and $62.5 \%$ in the evaluation of local recurrences, respectively.

The ${ }^{18} \mathrm{~F}$-FDG PET/CT detected 36 of 40 extrahepatic metastases correctly, thus lesion-based sensitivity, specificity, and accuracy were 90\%, 88.2\%, and 89.4\%, respectively. Serum CEA levels were elevated in 17 of 29 patients with extrahepatic metastases and normal values were detected in the remaining 12 patients, yielding false-negative results. The patient-basis sensitivity, specificity, and accuracy of CEA levels in the evaluation of extrahepatic metastases were calculated as 58.6\%, 64.7\%, and $60,8 \%$, respectively. CIM accurately detected 28 out of 40 
extrahepatic metastatic foci, whereas false-negative in 12 patients. Therefore, the lesion-based sensitivity, specificity, and accuracy were calculated as 70\%, 52.9\%, and 64.9\%, respectively.

Table 2 shows the comparative results of PET/CT, CIM, and serum CEA measurements.

Among the patients referred to ${ }^{18} \mathrm{~F}-\mathrm{FDG}$ PET/CT, increased CEA levels were found in 48 (64\%) patients and abnormal CIM findings in $53(71 \%)$ patients. All of the three modalities were truepositive in $28(49 \%)$ patients. In 13 (22\%) patients, ${ }^{18} \mathrm{~F}-\mathrm{FDG}$ PET/ CT and CEA measurements were true-positive, whereas CIM was false-negative. In 13 (22\%) patients, ${ }^{18} \mathrm{~F}$-FDG PET/CT and CIM were true-positive but CEA measurements were false-negative. The CIM correctly detected the recurrence in $3(5 \%)$ patients, whereas ${ }^{18}$ FDG PET/CT and CEA were false negatives. In 1 (2\%) patient, CIM and CEA measurements were true-positive, whereas the ${ }^{18} \mathrm{~F}-\mathrm{FDG}$ PET/CT was false-negative. No recurrence was detected in 3 patients referred with suspicious physical examination findings.

\section{DISCUSSION}

In our study, ${ }^{18} \mathrm{~F}$-FDG PET/CT correctly identified the recurrent disease in 54 out of 58 patients and yielded true-negative results in 15 of 17 patients without recurrent disease. Therefore, the sensitivity, specificity, accuracy, PPV, and NPV of ${ }^{18} \mathrm{~F}-\mathrm{FDG}$ PET/CT were found as $93.1 \%, 88.2 \%, 92 \%, 96.4 \%$, and $78.9 \%$, respectively. Thus, this study showed the superiority of ${ }^{18} \mathrm{~F}-\mathrm{FDG} \mathrm{PET} / \mathrm{CT}$ since these values were $72.4 \%, 64.7 \%, 70.6 \%, 87.5 \%, 40.7 \%$ and $77.5 \%$, $52.9 \%, 72 \%, 84.9 \%$, and $40.9 \%$ for serum CEA measurements and CIM, respectively.

One of 4 patients with false-negative results had normal serum tumor marker levels and was referred to PET/CT for the

\begin{tabular}{|c|c|c|c|}
\hline & $\mathrm{PET} / \mathrm{CT}$ & CIM & $\begin{array}{l}\text { Elevated serum } \\
\text { CEA levels }\end{array}$ \\
\hline Sensitivity (\%) & 93.1 & 77.5 & 72.4 \\
\hline Specificity (\%) & 88.2 & 52.9 & 64.7 \\
\hline PPV (\%) & 96.4 & 84.9 & 87.5 \\
\hline NPV (\%) & 78.9 & 40.9 & 40.7 \\
\hline Accuracy (\%) & 92 & 72 & 70.6 \\
\hline True-positive (n) & 54 & 45 & 42 \\
\hline True-negative (n) & 15 & 9 & 11 \\
\hline False-positive (n) & 2 & 8 & 6 \\
\hline False-negative (n) & 4 & 13 & 16 \\
\hline
\end{tabular}

evaluation of lung nodules detected with CT. No pathological FDG uptake in the nodules was observed on the PET/CT. The patient's clinical follow-up in the following months confirmed the suspicion of lung metastasis. This patient had rectal mucinous carcinoma, of which FDG affinity is low. The second patient who had undergone partial colectomy and liver metastasectomy for colon adenocarcinoma was referred to PET/ CT for investigation of the free fluid in the abdomen detected in the MRI. The free fluid in the abdomen showed no abnormal ${ }^{18} \mathrm{~F}-\mathrm{FDG}$ uptake on PET/CT imaging, but the investigation of ascites fluid revealed adenocarcinoma. The third patient with mucinous adenocarcinoma had a mass lesion in the rectus abdominis without ${ }^{18} \mathrm{~F}-\mathrm{FDG}$ uptake. The last patient with falsenegative showed no FDG uptake at the millimetric nodular lesions in the lungs probably due to the low affinity for FDG and/ or resolution limitations. On clinical follow-up, the lesions were found to be metastatic nodules in the lungs.

One of the patients with a false-positive result had a mass lesion in the presacral space, strongly suggestive of recurrence on PET/CT, but the histopathology revealed exudative material. In the second false-positive case, widespread abnormal FDG uptakes were found in the liver on PET/CT images, suggestive of metastasis but the histopathology revealed active chronic hepatitis.

Approximately, in two-thirds of patients with CRC, serum CEA levels are increased with a specificity of $70-84 \%(12,13)$. Rocklin et al. (14) and Carlsson et al. (15) showed in their studies that CEA itself alone is superior to other biochemical tests in detecting recurrences. Wanebo et al. (16) prospectively examined many laboratory and radiological studies used in the follow-up of patients with colon and rectal cancer, and they detected the recurrences with elevated levels of CEA in 89\% of patients. Serum CEA may show the recurrence earlier than all other diagnostic methods and symptoms (17). Serial CEA measurements are used in the follow-up of recurrence rather than clinical evaluation. However, the accuracy in the detection of locoregional recurrence and lung metastasis is not as high as it is in liver metastases (18). CIM can localize recurrences only 3-9 months after following the elevated CEA $(12,19)$.

The sensitivity, NPV, and accuracy of serum CEA in detecting liver metastases are found as $100 \%, 100 \%$, and $85 \%$, respectively. Meanwhile, the accuracy of serum CEA value in detecting local recurrence and extrahepatic metastasis is found as $70 \%$ and $60.8 \%$, respectively. The current study, as mentioned in the literature (18), shows that elevated values of serum CEA are highly indicative of liver metastases, whereas normal CEA does 
not rule out extrahepatic disease and local recurrence. In our study, CEA's NPV for local recurrence and extrahepatic metastases are calculated as $64.7 \%$ and $47.8 \%$, respectively.

In patients presenting with high serum CEA levels and presacral mass, CIM has difficulty in differentiating the recurrence from fibrotic/scar tissue (20). The ${ }^{18} \mathrm{~F}-\mathrm{FDG}$ PET/CT imaging not only has a very important role in distinguishing between local recurrence and scar tissue after surgery/radiotherapy but also allows the detection of unpredictable distant metastases $(21,22)$. The ${ }^{18}$ F-FDG PET/CT has a sensitivity of $90 \%$ and specificity of $94 \%$ in detecting pelvic recurrence (20). Huebner et al. (23) reported in their meta-analysis that ${ }^{18} \mathrm{~F}-\mathrm{FDG} \mathrm{PET} / \mathrm{CT}$ has a sensitivity of $97 \%$ and specificity of $76 \%$. In detecting local recurrences, the ${ }^{18}$ F-FDG PET/CT showed sensitivity, PPV, and NPV of 90\%, 88\%, and $92 \%$, respectively, whereas the sensitivity of CT/colonoscopy was reported as $71 \%$ (24).

In this study, all cases identified by the CIM as false positives consisted of postoperative fibrosis, scarring, and postradiotherapy changes. The CIM yielded false-negative results, especially for extrahepatic metastases. In the present study, ${ }^{18} \mathrm{~F}$-FDG PET/CT correctly identified all 23 local recurrences and showed sensitivity, NPV, and accuracy of $100 \%, 100 \%$, and 95\%, respectively (Figure 1). Serum CEA levels were elevated in 17 of 23 patients with confirmed local recurrences and false-negative in 6 cases, yielding 73.9\% sensitivity, $64.7 \%$ specificity, and 70\% accuracy. CIMs were true-positive in 16 of 23 patients and falsenegative in 7 patients, thus showing sensitivity, specificity, and accuracy of $69.5 \% 52.9 \%$, and $62.5 \%$, respectively. Our study results showed the superiority of ${ }^{18} \mathrm{~F}-\mathrm{FDG} \mathrm{PET} / \mathrm{CT}$ in detecting local recurrences following the literature findings.

When hematogenous dissemination is concerned, the primary site of metastasis for colorectal carcinoma is the liver (25). Liver metastases developed within 5 years in $50 \%$ of patients who had undergone curative surgery (26), and the only way to prolong the survey is by resecting the hepatic metastases (25). To be resectable, the hepatic metastases should be confined to one lobe, should be 1-4 in number, and without any other distant

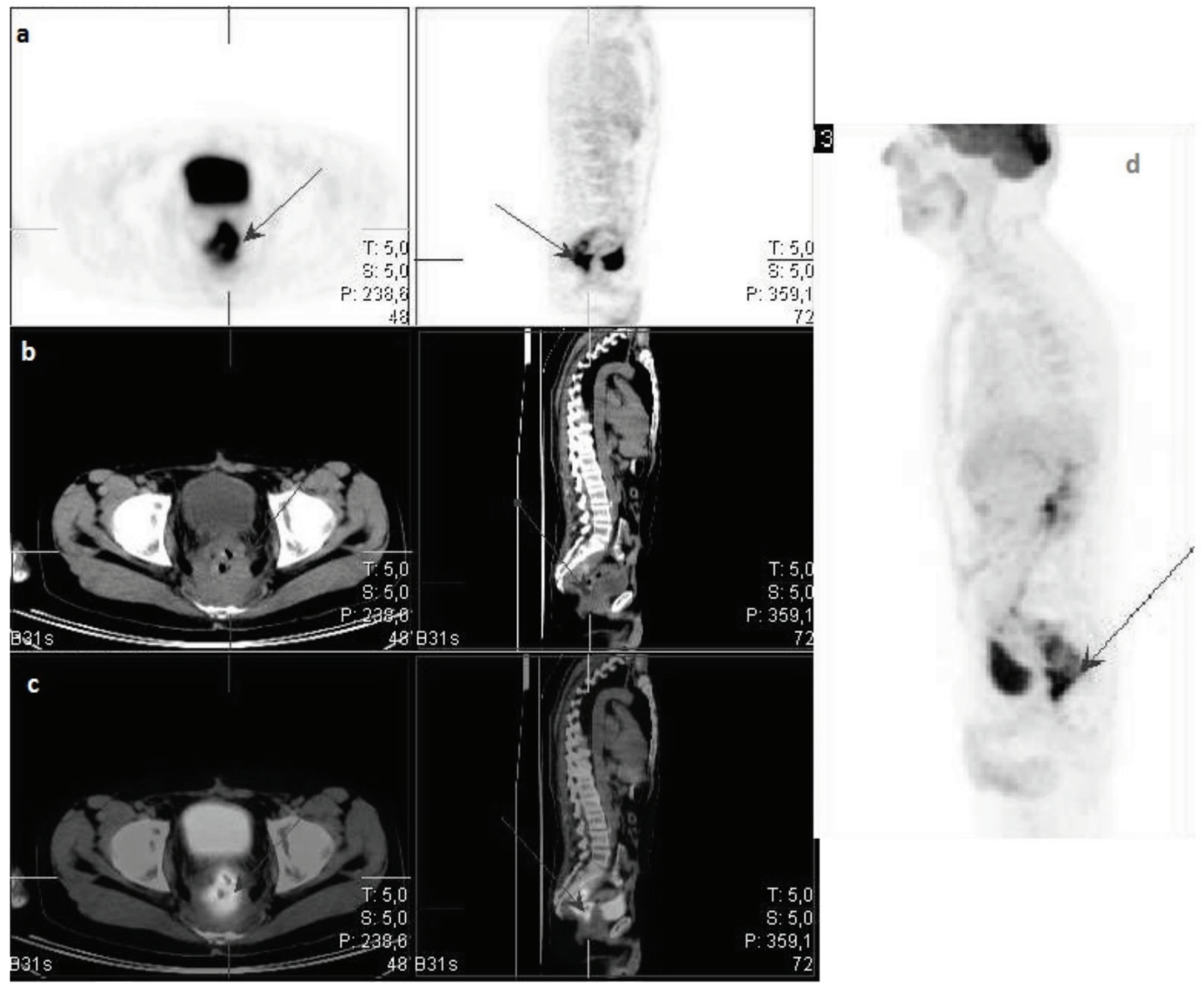

Figure 1. ${ }^{18} \mathrm{~F}$-FDG PET/CT images of a 51-year-old patient who had undergone a miles operation for colorectal cancer 5 years ago and was referred to $\mathrm{PET} / \mathrm{CT}$ for differentiation between scar tissue and local recurrence when CT detected a soft tissue mass at the presacral area. Serum CEA levels were normal. Axial PET (a), CT (b), fusion (c), and lateral MIP (d) images of the patient showed increased FDG uptake in the soft tissue mass at the presacral area consistent with metastasis (arrows)

${ }^{18} \mathrm{~F}-\mathrm{FDG}:{ }^{18} \mathrm{~F}$-fluorodeoxyglucose, PET/CT: Positron emission tomography/computed tomography, CEA: Carcinoembryonic antigen, MIP: Maximum intensity projection 
metastatic site or metastatic lymphadenopathy (27). Only 10$20 \%$ of the hepatic metastases could be candidates for surgical resection (28). After curative surgical excision, 5-year survival and 5-year disease-free survival were reported to be $33-40 \%$ and $22 \%$, respectively $(3,26)$. The underlying reason for this relatively short duration of disease-free survival is the presence of occult cancer foci that are not detected in patients initially believed to be candidates for curative resection. The ${ }^{18} \mathrm{~F}$-FDG PET/CT is an important imaging modality in terms of its high sensitivity in detecting liver metastases, as well as detecting unpredictable metastatic disease (25). In most patients who are planning to undergo partial liver resection, CT is used to detect any extrahepatic diseases. However, either extensive disease discovered during laparotomy or the recurrent hepatic or extrahepatic diseases emerging shortly after surgery during follow-up necessitates more effective presurgical imaging to prevent unnecessary surgery.

In our study, the ${ }^{18} \mathrm{FDG}$ PET/CT correctly identified all 24 liver metastases with a sensitivity, specificity, NPV, and accuracy of $100 \%, 88.2 \%, 100 \%$, and $95 \%$, respectively. In the same way, in all 24 patients with confirmed liver metastasis, serum CEA levels were higher with sensitivity, accuracy, and NPV of 100\%, $100 \%$, and 85\%, respectively. The CIM was true-positive in 19 out of 24 patients and false-negative in 5 patients yielding a sensitivity of $79 \%$ and accuracy of $68 \%$. Therefore, in our study, ${ }^{18} \mathrm{~F}$-FDG PET/CT showed a very high sensitivity in detecting liver metastases.

The PET/CT was found to be more sensitive than CIM in detecting extrahepatic metastases (94\% vs. 67\%) (24). Delbeke et al. (29) reported in their study that PET/CT had a sensitivity of $100 \%$ and accuracy of $92 \%$ in the demonstration of extrahepatic metastases, whereas these values for CT were $71 \%$ and $74 \%$, respectively. Peritoneal and mesenteric metastases with small volume are often missed with $\mathrm{CT}$, whereas ${ }^{18} \mathrm{~F}$-FDG PET/CT can easily detect these lesions unless they are very small $(<1 \mathrm{~cm})(29)$.

In the present study, the ${ }^{18} \mathrm{~F}-\mathrm{FDG}$ PET/CT correctly identified the extrahepatic metastases in 36 out of 40 lesions, showing a sensitivity of $90 \%$, specificity of $88.2 \%$, and accuracy of $89.4 \%$ (Figure 2). Serum CEA levels were high in 17 out of 29 patients with extrahepatic metastases and its sensitivity, specificity, and accuracy were found as $58.6 \%, 64.7 \%$, and $60.8 \%$, respectively. Meanwhile, CIM detected 28 out of 40 extrahepatic metastatic foci, thus yielding a sensitivity, specificity, and accuracy of $70 \%$, $52.9 \%$, and $64.9 \%$, respectively. In our study, the ${ }^{18} \mathrm{~F}$-FDG PET/CT's superiority to CIM was most prominent in detecting extrahepatic metastases.
All methods were true-positive in 28 (49\%) patients. Elevated serum CEA levels and positive ${ }^{18} \mathrm{~F}$-FDG PET/CT results were seen in 13 (22\%) patients, whereas CIM was false-negative in these patients. Contrarily, both ${ }^{18} \mathrm{~F}-\mathrm{FDG}$ PET/CT and CIM were truepositive in 13 (22\%) patients when CEA gave false-negative results. Therefore, ${ }^{18} \mathrm{~F}-\mathrm{FDG} \mathrm{PET} / \mathrm{CT}$ exhibited a $93 \%$ success rate in detecting recurrence in cases referred to ${ }^{18} \mathrm{~F}$-FDG PET/ CT depending on suspicious CIM findings and/or elevated CEA results.

The most important consequence of this high rate of metastases detection is changing the treatment protocol. Demonstrating unexpected metastatic lesions on ${ }^{18} \mathrm{~F}-\mathrm{FDG}$ PET/CT in patients who were assumed to have limited metastatic disease before metastasectomy might change the treatment protocol of patients with a ratio of $13-32 \%(21,29)$.

In our study, the ${ }^{18} \mathrm{~F}-\mathrm{FDG}$ PET/CT detected extrahepatic foci of metastases in 7 patients, thus 6 patients underwent chemotherapy instead of liver metastasectomy. In another patient with high CEA values, whose CIM was reported as normal, metastatic foci were found in the abdomen, and similarly, chemotherapy was initiated. Additionally, 3 out of 5 patients, whose liver metastases were not detected with CIM, underwent metastasectomy. In the other 2 patients, multiple metastases were detected in the liver, and chemotherapy was applied. In 7 patients with local recurrence, the ${ }^{18} \mathrm{~F}-\mathrm{FDG}$ PET/CT correctly identified the lesions, whereas CIM could not differentiate the lesions from postoperative fibrosis/scarring, and consequently 3 patients were scheduled for radiotherapy and 4 underwent surgery. In addition, 6 of 17 cases without the recurrent disease (except true-negative patients detected by CIM and PET/CT together), PET/CT yielded true-negative results and reported normal. Therefore, ${ }^{18} \mathrm{~F}$-FDG PET/CT has changed the management of patients in 25 out of 75 cases (33.3\%).

\section{CONCLUSION}

In conclusion, recurrent CRC was detected by ${ }^{18} \mathrm{FDG}$ PET/CT with high sensitivity and specificity as compared with the CIM. Our study results revealed the superiority of ${ }^{18} \mathrm{~F}$-FDG PET/CT in detecting local recurrences of CRC following the literature findings. The ${ }^{18} \mathrm{~F}$-FDG PET/CT showed a very high sensitivity in detecting liver metastases but its superiority to CIM was most prominent in detecting extrahepatic metastases. The ${ }^{18} \mathrm{~F}-\mathrm{FDG}$ $\mathrm{PET} / \mathrm{CT}$ has also substantially changed the management of patients. Our study revealed that CEA is highly sensitive in detecting liver metastases, but normal serum CEA levels do not rule out extrahepatic diseases and local recurrences. Recurrence 


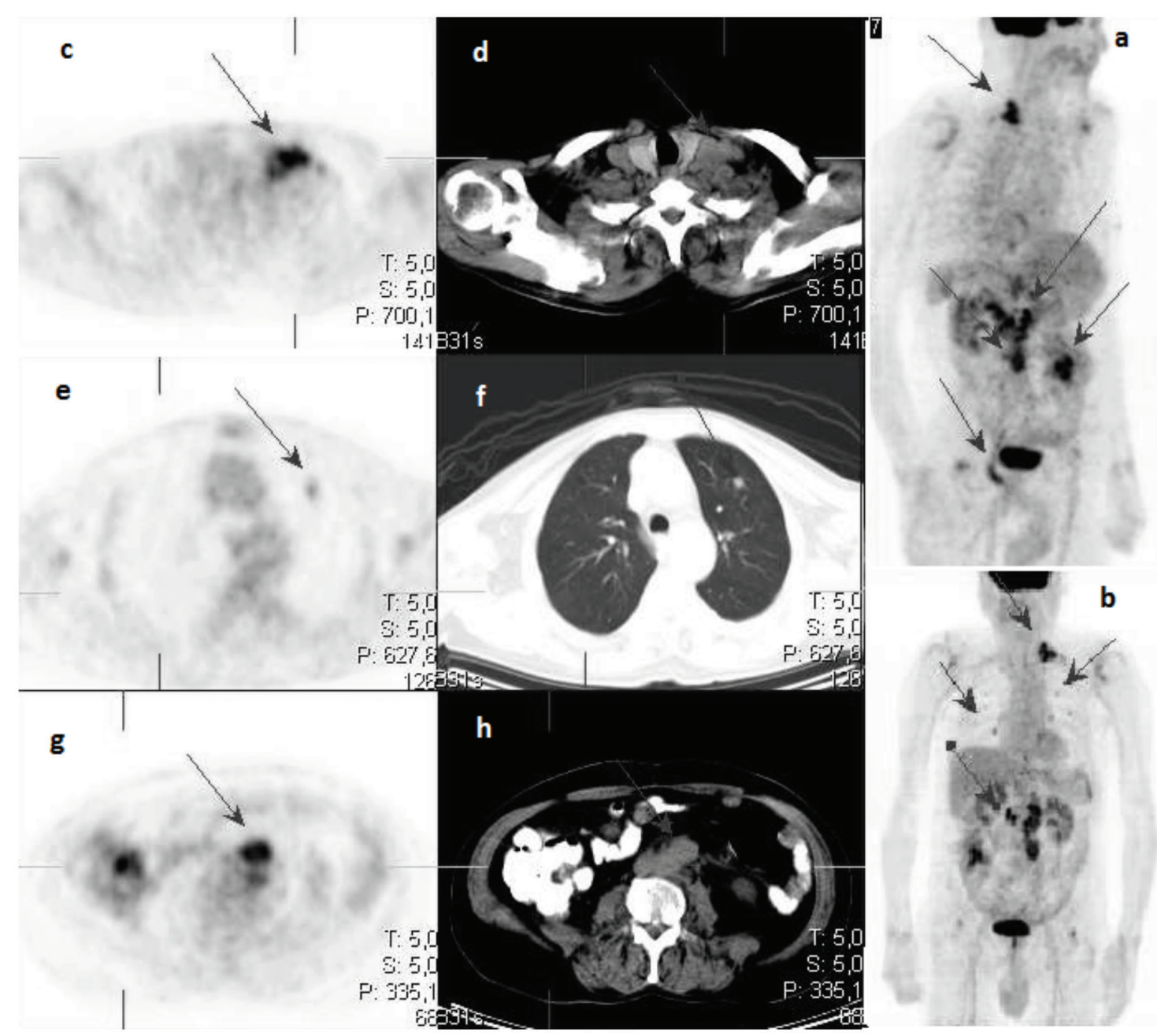

Figure 2. ${ }^{18} \mathrm{~F}$-FDG PET/CT images of a 74-year-old patient, who had undergone low anterior resection operation for rectal cancer 2 years ago and was referred to PET/CT for evaluation of the paraaortic lymph nodes detected with CT. Serum CEA levels were normal. Lateral (a) and anterior (b) MIP images showed pathologically increased FDG accumulations at the left supraclavicular region, nodules in both lungs, and intraabdominal paraaortic lymph nodes consistent with metastasis. Selected axial PET and CT images of the patient showed increased FDG uptake at the left supraclavicular region (c, d), at a pulmonary nodule in the left lung (e, f), and lymph nodes located in the left paraaortic region (g, h) (arrows)

${ }^{18} \mathrm{~F}-\mathrm{FDG}$ : ${ }^{18} \mathrm{~F}$-fluorodeoxyglucose, PET/CT: Positron emission tomography/computed tomography, MIP: Maximum intensity projection, CEA: Carcinoembryonic antigen

was detected by ${ }^{18} \mathrm{~F}-\mathrm{FDG}$ PET/CT imaging in $70.6 \%$ cases with elevated serum CEA levels and negative, equivocal and/or pathological findings of CIM.

The ${ }^{18} \mathrm{~F}-\mathrm{FDG}$ PET/CT exhibited a $93 \%$ success rate in detecting recurrences in cases referred to ${ }^{18} \mathrm{~F}-\mathrm{FDG} \mathrm{PET} / \mathrm{CT}$ depending on suspicious findings on CIM and/or elevated CEA results.

\section{Ethics}

Ethics Committee Approval: This study was carried out with the approval decision of Okmeydani Training and Research Hospital Clinical Research Ethics Committee dated April 20, 2009, and numbered 260.

Informed Consent: Consent was obtained from all patients who were scanned.
Peer-review: Externally peer-reviewed.

\section{Authorship Contributions}

Concept: A.K.U., T.Ö., Design: A.K.U., T.K.G., F.Ö., T.Ö., Data Collection or Processing: A.K.U., T.K.G., F.Ö., Analysis or Interpretation: A.K.U., Literature Search: A.K.U., T.K.G., F.Ö., T.Ö., Writing: A.K.U.

Conflict of Interest: No conflict of interest was declared by the authors.

Financial Disclosure: The authors declared that this study received no financial support. 


\section{REFERENCES}

1. Jemal A, Siegel R, Ward E, Hao Y, Xu J, Thun MJ. Cancer statistics 2009. CA Cancer J Clin 2009;59:225-49.

2. Flamen P, Stroobants S, Van Cutsem E, Dupont P, Bormans G, De Vadder $\mathrm{N}$, et al. Additional value of whole-body positron emission tomography with fluorine-18-2-fluoro-2-deoxy-D-glucose in recurrent colorectal cancer. J Clin Oncol 1999;17:894-901.

3. Kalff V, Hicks RJ, Ware RE, Hogg A, Binns D, McKenzie AF. The clinical impact of (18)F-FDG PET in patients with suspected or confirmed recurrence of colorectal cancer: a prospective study. J Nucl Med 2002:43:492-9.

4. Tepper JE, O'Connell M, Hollis D, Niedzwiecki D, Cooke E, Mayer RJ, et al. Analysis of surgical salvage after failure of primary therapy in rectal cancer: results from Intergroup Study 0114. J Clin Oncol 2003;21:36238.

5. Goldberg RM, Fleming TR, Tangen CM, Moertel CG, Macdonald JS, Haller DG, et al. Surgery for recurrent colon cancer: strategies for identifying resectable recurrence and success rates after resection. Eastern Cooperative Oncology Group, the North Central Cancer Treatment Group, and the Southwest Oncology Group. Ann Intern Med 1998;129:27-35.

6. Chong G, Cunningham D. Gastrointestinal cancer: recent developments in medical oncology. Eur J Surg Oncol 2005;31:453-60.

7. Punt CJ. New options and old dilemmas in the treatment of patients with advanced colorectal cancer. Ann Oncol 2004;15:1453-9.

8. Bennett JJ, Cao D, Posner MC. Determinants of unresectability and outcome of patients with occult colorectal hepatic metastases. J Surg Oncol 2005;92:64-9.

9. Schiepers C, Penninckx F, De Vadder N, Merckx E, Mortelmans L, Bormans $\mathrm{G}$, et al. Contribution of PET in the diagnosis of recurrent colorectal cancer: comparison with conventional imaging. Eur J Surg Oncol 1995;21:517-22.

10. Zervos EE, Badgwell BD, Burak WE Jr, Arnold MW, Martin EW. Fluorodeoxyglucose positron emission tomography as an adjunct to carcinoembryonic antigen in the management of patients with presumed recurrent colorectal cancer and nondiagnostic radiologic workup. Surgery 2001;130:636-43; discussion 643-4.

11. Sarikaya I, Bloomston M, Povoski SP, Zhang J, Hall NC, Knopp MV, et al. FDG-PET scan in patients with clinically and/or radiologically suspicious colorectal cancer recurrence but normal CEA. World J Surg Oncol 2007;5:64.

12. Moertel CG, Fleming TR, Macdonald JS, Haller DG, Laurie JA, Tangen C. An evaluation of the carcinoembryonic antigen (CEA) test for monitoring patients with resected colon cancer. JAMA 1993;270:943-7.

13. Fletcher RH. Carcinoembryonic antigen. Ann Intern Med 1986;104:6673.

14. Rocklin MS, Senagore AJ, Talbott TM. Role of carcinoembryonic antigen and liver function tests in the detection of recurrent colorectal carcinoma. Dis Colon Rectum 1991;34:794-7.
15. Carlsson U, Stewénius J, Ekelund G, Leandoer L, Nosslin B. Is CEA analysis of value in screening for recurrences after surgery for colorectal carcinoma? Dis Colon Rectum 1983;26:369-73.

16. Wanebo HJ, Llaneras M, Martin T, Kaiser D. Prospective monitoring trial for carcinoma of colon and rectum after surgical resection. Surg Gynecol Obstet 1989;169:479-87.

17. Graham RA, Wang S, Catalano PJ, Haller DG. Postsurgical surveillance of colon cancer: preliminary cost analysis of physician examination, carcinoembryonic antigen testing, chest $\mathrm{x}$-ray, and colonoscopy. Ann Surg 1998;228:59-63.

18. Esteves FP, Schuster DM, Halkar RK. Gastrointestinal tract malignancies and positron emission tomography: an overview. Semin Nucl Med 2006;36:169-81.

19. McCall JL, Black RB, Rich CA, Harvey JR, Baker RA, Watts JM, et al. The value of serum carcinoembryonic antigen in predicting recurrent disease following curative resection of colorectal cancer. Dis Colon Rectum 1994;37:875-81.

20. Tzimas GN, Koumanis DJ, Meterissian S. Positron emission tomography and colorectal carcinoma: an update. J Am Coll Surg 2004;198:645-52.

21. Delbeke D. Oncological applications of FDG PET imaging: brain tumors, colorectal cancer, lymphoma and melanoma. J Nucl Med 1999;40:591603.

22. Willkomm P, Bender H, Bangard M, Decker P, Grünwald F, Biersack HJ. FDG PET and immunoscintigraphy with 99mTc labeled antibody fragments for detection of recurrence of colorectal carcinoma. J Nucl Med 2000;41:1657-63.

23. Huebner RH, Park KC, Shepherd JE, Schwimmer J, Czernin J, Phelps ME, et al. A meta-analysis of the literature for whole-body FDG PET detection of recurrent colorectal cancer. J Nucl Med 2000;41:1177-89.

24. Whiteford MH, Whiteford HM, Yee LF, Ogunbiyi OA, Dehdashti F, Siegel $B A$, et al. Usefulness of FDG-PET scan in the assessment of suspected metastatic or recurrent adenocarcinoma of the colon and rectum. Dis Colon Rectum 2000;43:759-67; discussion 767-70.

25. Rydzewski B, Dehdashti F, Gordon BA, Teefey SA, Strasberg SM, Siegel BA. Usefulness of intraoperative sonography for revealing hepatic metastases from colorectal cancer in patients selected for surgery after undergoing FDG PET. AJR Am J Roentgenol 2002;178:353-8.

26. Ruers TJ, Langenhoff BS, Neeleman N, Jager GJ, Strijk S, Wobbes T, et al. Value of positron emission tomography with [F-18]fluorodeoxyglucose in patients with colorectal liver metastases: a prospective study. J Clin Oncol 2002;20:388-95.

27. Thoeni RF. Colorectal cancer. Radiologic staging. Radiol Clin North Am 1997;35:457-85.

28. Lee FT Jr, Mahvi DM, Chosy SG, Onik GM, Wong WS, Littrup PJ, et al. Hepatic cryosurgery with intraoperative US guidance. Radiology 1997;202:624-32.

29. Delbeke D, Vitola JV, Sandler MP, Arildsen RC, Powers TA, Wright JK Jr, et al. Staging recurrent metastatic colorectal carcinoma with PET. J Nucl Med 1997;38:1196-201. 